\title{
Making the best of an unappealing situation
}

\section{The appeals process allows authors to ask us to reconsider a negative decision. Here we provide advice on when it's worth appealing and what makes a successful appeal.}

$\triangle 1$ fter months, even years, of hard work, you've carefully written and submitted your manuscript to NSMB. What follows is a brief respite mixed with hope that your paper will be sent out to review. Suddenly, the moment of truth arrives_-you've got mail from NSMB. The next thing you know, your hard work has been met with something that nobody in any walk of life likes to receive: rejection. How could NSMB have missed the importance of these results and the interest that this work will garner? If the paper was sent out to review, you've waited for at least two weeks, only to find out that the reviewers want a long list of additional experiments before they'll give it the thumbs up. How could they not be satisfied with the work you've already done? These are all reasonable responses; the question is what to do next.

Our October 2006 editorial explained that when you receive the rejection letter, the paper and reviews (if present) have already been discussed among the editors. We receive a huge volume of submissions, $75-80 \%$ of which do not even reach review; we cannot publish everything. As we are human and can make mistakes, there is an official avenue for asking us to reconsider our decision: an unwieldy beast called the appeal.

\section{Should I appeal the decision?}

Appeals are rarely successful and take considerable time, so you should be fairly sure that you have solid grounds for one. It can take up to two weeks to receive an editorial decision after lodging an appeal, not because we are peevish or do not take them seriously, but because they take second place to our normal workload. Also, fully considering an appeal involves rereading the manuscript, reviews if present, the points raised in the appeal and other relevant papers. The appeal is then discussed among all editors before a decision is reached. A frivolous appeal will thus inevitably cost you time, time the paper could have spent in review at an alternative journal.

\section{How soon should I appeal a decision?}

Try not to have a knee-jerk reaction, and don't fire off an appeal e-mail within minutes of receiving the decision letter. Take time to consider what the decision letter and reviews (if present) say, and carefully assess whether you have a sound basis for an appeal. But do not wait too long: editorial folklore tells of a paper that disappeared into the ether for a year (read, 'into the submission systems of other journals') and came back with Figure 6 and Figure 1 exchanged. If the appeal comes after a long time lag, we look for additional experimentation addressing the editors' and (if appropriate) reviewers' concerns_-simply reshuffling or rewording a manuscript will not do it.

What makes an effective appeal?

There are more and less effective ways of appealing. Perhaps unsurprisingly, the former correlates with an 'objective' appeal (based on cold, hard scientific facts and argument) and the latter with more 'subjective' appeals ('I was shocked by your decision', 'Dr. Z thinks this should be published', 'They liked my talk at meeting Y').

\section{Which appeals are most successful?}

Those that introduce new data directly addressing the editorial and/or reviewer concerns, or that contact us asking: 'If I add these data or expand this aspect of the paper, will you consider the revised manuscript?'

Which appeals are least successful?

Without a doubt, those that are trying to convince us of interest. This is not impossible: it could be that the message of the paper was buried and we missed it. But citing all the Science and Nature papers in the field for the last ten years will not make your paper fit our scope or make the particular advance described in the paper more interesting to our broad readership. Many of the subjective-type statements above arise when authors are trying to convince us of interest.

Do the editors alone decide the outcome of an appeal?

Generally, yes. For appeals after review, we will sometimes consult the reviewers on whether they find your arguments convincing or whether the proposed experiments will adequately address the important issues. For appeals based on field controversy or interest, we will sometimes consult someone to get an outside opinion.

What if I think one of the reviewers was unfair?

We have had appeals that tell us that a reviewer seems biased or inappropriate when the author suggested the reviewer in question! If there is something apparently inaccurate or biased in the review, alert us and we may consult one of the other reviewers on the particular point being made. Rest assured that the editors are aware if one reviewer is vitriolic or out of step with the others. In extreme circumstances, we will engage an additional reviewer (but a new reviewer can make new suggestions that the editors may want the authors to consider).

Any nuts-and-bolts advice for writing the appeal?

The appeal should respond to the concerns raised by editors and/or reviewers and should explain clearly and up front how these concerns can be addressed. It doesn't hurt to be polite. It doesn't help to insult the editors, the journal or the reviewers. Some appeals have started with three paragraphs of diatribe against the journal followed by a suggestion as to how the paper will be revised for reconsideration. We do read appeal letters carefully, but it's best not to take a risk by burying important points in this way.

And finally...

It is important to keep in mind that, when used effectively, the appeal can be positive for everyone concerned. It gives you, the author, a chance to better make your case to the editors and the reviewers, and if all goes well, the end result is an improved paper published in NSMB. Even the disappointment of a second negative decision can ultimately lead to a better understanding of what makes a manuscript a strong candidate at NSMB, thus increasing the chances that future submissions will be successful. 